\title{
Synthesis, Characterizations and Biological Screening of Tetrahydro-Quinazoline Analogues
}

\author{
Hiren Doshi ${ }^{1{ }^{* *},}$ Maitreya Bhatt ${ }^{1}$, Sampark Thakkar $^{2}$, Arabinda Ray ${ }^{2}$ \\ ${ }^{1}$ Ashok \& Rita Patel Institute of Integrated Study \& Research in Biotechnology and Allied Sciences (ARIBAS), Sardar Patel University, \\ NewV.V.Nagar, 388121, Gujarat, India \\ ${ }^{2}$ P. D. Patel Institute of Applied Sciences (PDPIAS), CHARUSAT, Changa, 388421, Gujarat, India
}

\begin{abstract}
Quinazoline and their fused-ring systems are well known for their potential biological activity. In the present study a new Tetrahydro-quinazoline analogues (MB I-V) were synthesized. The newly synthesized compounds were characterized by IR, NMR and C, H, N, S analyses. All newly synthesized compounds were screened for their antibacterial (Pseudomonas aurigenosa Bacillus subtilis and Escherichia coli) studies. The results revealed that all synthesized compounds have a significant biological activity against the tested microorganisms.
\end{abstract}

Keywords Tetrahydro-Quinazoline, Antibacterial Activity, Isophorone

\section{Introduction}

During the ancient era the isolation of various compounds was done by the process of extraction. But this process was time consuming as well as laborious. Moreover the yield was very low and and the process of isolation required large amount of the starting material. Today the process of isolation has been replaced by the synthetic routes. A large number of compounds can be synthesized by using small amount of chemicals. More over the Synthetic routes take less amounts of time and can easily be carried out. Quinazoline derivatives hold a place of significant in todays world for their important application in chemical, clinical and biological spheres. Medicinally quinazoline has been used in various areas especially as an analgesic[1, 2], anti-oxidant[3-5], anti-cancer drugs[6-10], anti-inflammator $\mathrm{y}[11,12], \quad$ anti-convulsant[13], anti-bacterial[14], anti-fungal[15] and anti-mycobacterial agents[16, 17]. It has also been found in the treatment of malaria[18, 19]. Considering the vast potential of quinazoline, it was thought appropriate to synthesized, characterized quinazoline analogues and investigates their biological activity. In this investigation, we have prepared tetrahydro quinazoline analogues and characterized them using spectral data. Biological screenings of these compounds were also reported also reported here.

* Corresponding author:

drhirendoshi@yahoo.co.in (Hiren Doshi)

Published online at http://journal.sapub.org/ajoc

Copyright (C) 2012 Scientific \& Academic Publishing. All Rights Reserved

\section{Result and Discussion}

\subsection{Chemistry}

Intermediates, 5,5,7-trimethyl-4-aryl-3,4,5,6-tetrahydro quinazoline -2-thiols (S I-V) synthesized from isophorone, different aromatic aldehydes and thiourea (Scheme Ia,b). Tetrahydroquinazoline analogues (MB I-V) synthesized from intermediates S I-V (Scheme IIa,b). The purity of the compounds was monitored by ascending thin layer chromatography (TLC) on silica gel-G coated on aluminum plates.

Developing solvents used in TLC were ethyl acetate: hexane (1:2) and the plates were viewed under UV light 254 $\mathrm{nm}$ and $265 \mathrm{~nm}$ respectively. Melting points were determined by the open capillary tubes equipped in electro thermal melting point apparatus. All the melting points are uncorrected. The yields of all the compounds reported are of crystallized form. The elemental analysis was studied by C, H, N, S analyzer on Perkin Elmer (U.S.A, 2400 Series II). Infrared (IR) spectra were recorded for the compounds on Perkin Elmer Spectrum GX using KBr pellet disc technique. The structure will further be elucidated by recording its mass spectra on Shimadzu LCMS $2010 \mathrm{eV}$. NMR was recorded in Torrent pharmaceuticals Ltd Research centre on Bruker Avance FT- NMR $400 \mathrm{MHz}$.

The M.P. of synthesized compounds were found between $258-300^{\circ} \mathrm{C}$, while for the some analogues it was more than $300^{\circ} \mathrm{C}$. The mol. wt. of the synthesized compound were in range of 271-427 $\mathrm{g} \mathrm{mole}^{-1}$. 


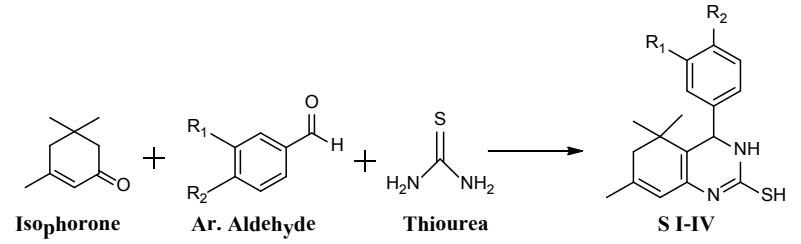

$\begin{array}{ccc}\text { Sample ID } & \mathrm{R}_{1} & \mathrm{R}_{2} \\ \text { S I } & -\mathrm{OH} & -\mathrm{OCH}_{3} \\ \text { S II } & -\mathrm{NO}_{2} & \mathrm{H} \\ \text { S III } & \mathrm{H} & \mathrm{H} \\ \text { S IV } & -\mathrm{OCH}_{3} & -\mathrm{OCH}_{3}\end{array}$

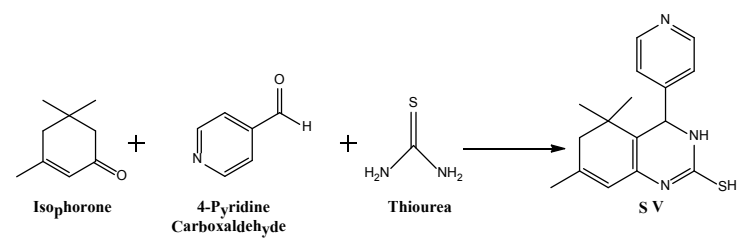

Scheme Ib
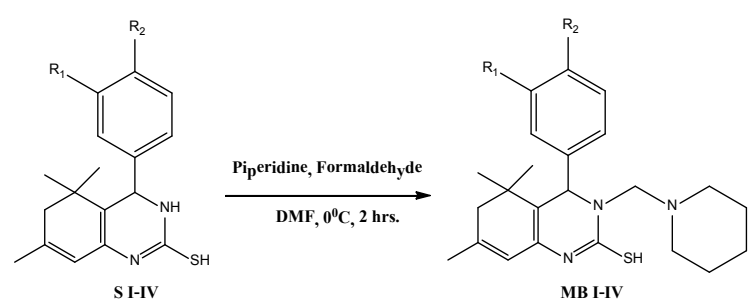

MB I-IV

$\begin{array}{ccc}\text { Sample ID (II) } & \mathrm{R}_{1} & \mathrm{R}_{2} \\ \text { MB I } & -\mathrm{OH} & -\mathrm{OCH}_{3} \\ \text { MB II } & -\mathrm{NO}_{2} & \mathrm{H} \\ \text { MB III } & \mathrm{H} & \mathrm{H} \\ \text { MB IV } & -\mathrm{OCH}_{3} & -\mathrm{OCH}_{3}\end{array}$

Scheme IIa



Scheme IIb

The IR peaks obtained in study was correlated with the standard IR frequency of the vibration mode given by Nakamoto[20]. The IR spectra showed the presence of $\mathrm{C}=\mathrm{C}$ stretching between $1580-1615 \mathrm{~cm}^{-1}, \mathrm{C}-\mathrm{C}$ stretching between 1030-1350 $\mathrm{cm}^{-1}, \mathrm{C}-\mathrm{H}$ stretching at $\sim 3080 \mathrm{~cm}^{-1}, \mathrm{C}-\mathrm{O}$ stretching $\left(1^{\circ}\right.$ alcohol $)$ at $1123 \mathrm{~cm}^{-1}, \mathrm{C}=\mathrm{N}$ stretching between $1080-1360 \mathrm{~cm}^{-1}$, N-H stretching between $3380-3400 \mathrm{~cm}^{-1}$, C-N stretching between $1150-1210 \mathrm{~cm}^{-1}$, C-S stretching between $685-710 \mathrm{~cm}^{-1},-\mathrm{NO}_{2}$ stretching at $\sim 1528 \mathrm{~cm}^{-1}$, -OH stretching at $\sim 3426 \mathrm{~cm}^{-1}$ for intermediates $\mathrm{S} \mathrm{I-V}$ and final products MB I-V. In addition, ${ }^{1 \mathrm{H}} \mathrm{NMR}$ spectra of the synthesized compounds showed the characteristic signals at $\sim 6.1 \delta$ due to $\mathrm{SH}$ group. Further the spectra of the intermediates (S I-V) showed a signal $\sim 4.1 \delta$ due to $\mathrm{NH}$ of pyrimidine ring and disappearance of these signals in synthesized compounds (MB I-V) established that attachment of piperidine moiety with pyrimidine $\mathrm{NH}$ group. The structures of synthesized compounds were supported by mass spectrum; it showed the molecular ion peaks in between range of $285-428(\mathrm{M}+1)$.

\subsection{Antibacterial Activity}

In present study the five compounds are screen for antibacterial activity against gram positive and gram negative bacteria. The result of antibacterial activities are comparatively studied (Table 1) and are recorded on the basis of the presence or absence of inhibition zone on media plate. The antibacterial activity against Bacillus subtilis was seen in all the compounds (MB I-V) used. The compound MB I showed high activity against Bacillus subtilis due to presence of OH group. All the compounds (MB I-V) did not show activity on Pseudomonas aurigenosa as well as the standard drug did not show any activity. The compound MB II showed high activity against Escherichia coli. This could be due to the presence of $-\mathrm{NO}_{2}$ group at $\mathrm{R}_{1}$ position of tetrahydroquinazoline derivatives.

Table 1. Zone of growth inhibition of synthesized compounds

\begin{tabular}{|c|c|c|c|}
\hline \multirow{2}{*}{ Sample } & \multicolumn{3}{|c|}{ Zone of Inhibition in mm } \\
\cline { 2 - 4 } & Bacillus subtilis & $\begin{array}{c}\text { Pseudomonas } \\
\text { aurigenosa }\end{array}$ & Escherichia coli \\
\hline MB I & 23 & - & 17 \\
\hline MB II & 22 & - & 23 \\
\hline MB III & 21 & - & 20 \\
\hline MB IV & 18 & - & 21 \\
\hline MB V & 16 & - & 15 \\
\hline Ciprofloxacin & 46 & - & 20 \\
\hline DMSO & - & - & - \\
\hline
\end{tabular}

This activity of any compound is directly correlated with their zone of inhibition on the medium. More than $20 \mathrm{~mm}$ of inhibition zone is called high activity, More than $10 \mathrm{~mm}$ of inhibition is called good activity, inhibition zone between 6-9 $\mathrm{mm}$ moderate activity and least activity is assign between 1-5 $\mathrm{mm}$ of inhibition zone[21].

\section{Experimental}

The raw materials used were of synthetic grade from Sigma Aldrich \& Loba chemicals Ltd. Solvents used were distilled and dried. The TLC plates were from Merck. Melting points were determined by the open capillary tubes equipped reported are of crystallized form in electro thermal melting point apparatus. All the melting points are uncorrected. The elemental analysis was studied by $\mathrm{C}, \mathrm{H}, \mathrm{N}$, S analyzer on Perkin Elmer (U.S.A, 2400 Series II). Infrared (IR) spectra were recorded for the compounds on Perkin Elmer Spectrum GX using KBr pellet disc technique. The structure will further be elucidated by recording its mass spectra on Shimadzu LCMS $2010 \mathrm{eV}$. NMR was recorded in 
Torrent pharmaceuticals Ltd Research centre on Bruker Avance FT- NMR 400 MHz.

\subsection{General Procedure for Synthesis of 5, 5, 7-Trimethyl -4-Aryl-3, 4, 5, 6-Tetrahydroquinazoline-2-Thios (S I-V)}

Equivalent mixture of isophorone, an aromatic aldehydes and thiourea was refluxed in ethanol for 10 hours. Then the resulting reaction mixture was poured in ice bath. The obtain product (Scheme-Ia \& Ib) was filtered, dried \& recrystallized by $95 \%$ Ethanol[20].

5-(2-mercapto-5,5,7-trimethyl-3,4,5,6-tetrahydroquinazol in-4-yl)-2-methoxyphenol (S I): Yield: 85\%; Colour: Brown; m.p.: $>300^{\circ} \mathrm{C}$; IR $\left(\mathrm{KBr}, \mathrm{cm}^{-1}\right)$ : $1462(\mathrm{Ar} \mathrm{C}=\mathrm{C}), 1598(\mathrm{Ar}$ C-C), $3403(\mathrm{~N}-\mathrm{H}), 3292(\mathrm{O}-\mathrm{H}), 1365(\mathrm{CH} 3), 1638(\mathrm{C}=\mathrm{N})$, 1219 (C-S), 1274 (Ester C-O); ${ }^{1} \mathrm{H}$ NMR (DMSO): $1.25 \delta(6 \mathrm{H}$, $\mathrm{s}, \mathrm{CH} 3), 1.83 \delta(3 \mathrm{H}, \mathrm{s}, \mathrm{CH} 3), 2.19 \delta(2 \mathrm{H}, \mathrm{s}, \mathrm{CH} 2), 3.83 \delta(3 \mathrm{H}$, s, OCH3), $4.28 \delta(1 \mathrm{H}, \mathrm{d}, \mathrm{N}-\mathrm{H}), 4.60 \delta(1 \mathrm{H}, \mathrm{d}, \mathrm{CH}), 5.35 \delta$ (1H. s, OH), $5.7 \delta(1 \mathrm{H}, \mathrm{s}, \mathrm{CH}), 6.3 \delta(1 \mathrm{H}, \mathrm{s}, \mathrm{S}-\mathrm{H}), 6.68 \delta(1 \mathrm{H}$, $\mathrm{d}, \mathrm{CH}), 6.70 \delta(1 \mathrm{H}, \mathrm{d}, \mathrm{CH}), 6.86 \delta(1 \mathrm{H}, \mathrm{s}, \mathrm{CH}), \mathrm{DMSO} 2.52 \delta$ \& $3.50 \delta$; MS, $\mathrm{m} / \mathrm{z}: 317.34[\mathrm{M}+1]$. Anal.: Calc. for $\mathrm{C}, 65.42$; H, 6.71; N, 8.48; S, 9.70. Found: C, 65.21; H, 6.50; N, 8.29; S, 9.35 .

5,5,7-trimethyl-4-(3-nitrophenyl)-3,4,5,6-tetrahydroquina zoline-2-thiol (S II): Yield: 78\%; Colour: Brown; m.p.: $>300^{\circ} \mathrm{C}$; IR $\left(\mathrm{KBr}, \mathrm{cm}^{-1}\right)$ : $1664(\mathrm{Ar} \mathrm{C}=\mathrm{C}), 1468(\mathrm{Ar}$ $\mathrm{C}-\mathrm{C}), 3383(\mathrm{~N}-\mathrm{H}), 3040(\mathrm{Ar} \mathrm{C}-\mathrm{H}), 1384\left(\mathrm{CH}_{3}\right), 1619(\mathrm{C}=\mathrm{N})$, 1246 (C-S), 1528 (N-O); ${ }^{1} \mathrm{H}$ NMR (DMSO): $1.26 \delta(6 \mathrm{H}, \mathrm{s}$, $\left.\mathrm{CH}_{3}\right), 1.82 \delta\left(3 \mathrm{H}, \mathrm{s}, \mathrm{CH}_{3}\right), 1.98 \delta\left(2 \mathrm{H}, \mathrm{s}, \mathrm{CH}_{2}\right), 4.12 \delta(1 \mathrm{H}, \mathrm{d}$, $\mathrm{N}-\mathrm{H}), 4.59 \delta(1 \mathrm{H}, \mathrm{d}, \mathrm{CH}), 5.61 \delta(1 \mathrm{H}, \mathrm{s}, \mathrm{CH}), 5.8 \delta(1 \mathrm{H}, \mathrm{s}$, S-H), $7.62 \delta(1 \mathrm{H}, \mathrm{d}, \mathrm{CH}), 7.59 \delta(1 \mathrm{H}, \mathrm{d}, \mathrm{CH}), 8.07 \delta(1 \mathrm{H}, \mathrm{d}$, $\mathrm{CH}), 8.12 \delta(1 \mathrm{H}, \mathrm{s}, \mathrm{CH})$, DMSO $2.48 \delta \& 3.47 \delta ; \mathrm{MS}, \mathrm{m} / \mathrm{z}$ : 329.23[M+1]. Anal.: Calc. for C, 61.98; H, 5.81; N, 12.76; S, 9.73. Found: C, $61.75 ; \mathrm{H}, 5.75 ; \mathrm{N}, 12.50 ; \mathrm{S}, 9.60$.

5,5,7-trimethyl-4-phenyl-3,4,5,6-tetrahydroquinazoline-2 -thiol (S III): Yield: 86\%; Colour: Brown; m.p.: $258^{\circ} \mathrm{C}$; IR (KBr, cm-1): IR ( KBr, cm $\left.{ }^{-1}\right): 1663(\mathrm{Ar} \mathrm{C}=\mathrm{C}), 1452(\mathrm{Ar} \mathrm{C}-\mathrm{C})$, $3438(\mathrm{~N}-\mathrm{H}), 2848(\mathrm{Ar} \mathrm{C}-\mathrm{H}), 1378\left(\mathrm{CH}_{3}\right), 1617(\mathrm{C}=\mathrm{N}) 1286$ (C-S); ${ }^{1} \mathrm{H}$ NMR (DMSO): $1.24 \delta\left(6 \mathrm{H}, \mathrm{s}, \mathrm{CH}_{3}\right), 1.83 \delta(3 \mathrm{H}, \mathrm{s}$, $\left.\mathrm{CH}_{3}\right), 2.2 \delta\left(2 \mathrm{H}, \mathrm{s}, \mathrm{CH}_{2}\right), 4.01 \delta(1 \mathrm{H}, \mathrm{d}, \mathrm{N}-\mathrm{H}), 4.62 \delta(1 \mathrm{H}, \mathrm{d}$, $\mathrm{CH}), 5.68 \delta(1 \mathrm{H}, \mathrm{s}, \mathrm{CH}), 5.9 \delta(1 \mathrm{H}, \mathrm{s}, \mathrm{S}-\mathrm{H}), 7.25 \delta(2 \mathrm{H}, \mathrm{t}$, $\mathrm{CH}), 7.28 \delta(1 \mathrm{H}, \mathrm{t}, \mathrm{CH}), 7.34 \delta(2 \mathrm{H}, \mathrm{t}, \mathrm{CH}), \mathrm{DMSO} 2.52 \delta \&$ $3.53 \delta ; \mathrm{MS}, \mathrm{m} / \mathrm{z}: 284.10[\mathrm{M}+1]$. Anal.: Calc. for C, $71.79 ; \mathrm{H}$, 7.09; N, 9.85; S, 11.27. Found: C, 71.50; H, 6.95; N, 9.67 S, 11.01 .

4-(3,4-dimethoxyphenyl)-5,5,7-trimethyl-3,4,5,6-tetrahyd roquinazoline-2-thiol (S IV): Yield: 91\%; Colour: Orange; m.p.: $285^{\circ} \mathrm{C}$; IR $\left(\mathrm{KBr}, \mathrm{cm}^{-1}\right)$ : $1652(\mathrm{Ar} \mathrm{C}=\mathrm{C}), 1462(\mathrm{Ar} \mathrm{C}-\mathrm{C})$, $3192(\mathrm{~N}-\mathrm{H}), 2955(\mathrm{Ar} \mathrm{C}-\mathrm{H}), 1381\left(\mathrm{CH}_{3}\right), 1612(\mathrm{C}=\mathrm{N}), 1249$ (C-S); ${ }^{1} \mathrm{H}$ NMR (DMSO): $1.26 \delta\left(6 \mathrm{H}, \mathrm{s}, \mathrm{CH}_{3}\right), 1.82 \delta(3 \mathrm{H}, \mathrm{s}$, $\left.\mathrm{CH}_{3}\right), 2.20 \delta\left(2 \mathrm{H}, \mathrm{s}, \mathrm{CH}_{2}\right), 3.88 \delta\left(6 \mathrm{H}, \mathrm{s}, \mathrm{OCH}_{3}\right), 4.11 \delta(1 \mathrm{H}$, $\mathrm{d}, \mathrm{N}-\mathrm{H}), 4.59 \delta(1 \mathrm{H}, \mathrm{d}, \mathrm{CH}), 5.69 \delta(1 \mathrm{H}, \mathrm{s}, \mathrm{CH}), 6.01 \delta(1 \mathrm{H}, \mathrm{s}$, $\mathrm{S}-\mathrm{H}), 6.76 \delta(1 \mathrm{H}, \mathrm{d}, \mathrm{CH}), 6.67 \delta(1 \mathrm{H}, \mathrm{d}, \mathrm{CH}), 6.73 \delta(1 \mathrm{H}, \mathrm{s}$, $\mathrm{CH})$, DMSO $2.5 \delta \& 3.51 \delta$; MS, m/z: $344.40[\mathrm{M}+1]$. Anal.: Calc. for C, 66.25; H, 7.02; N, 8.13; S, 9.31. Found: C, 65.99; H, 6.88; N, 8.04; S, 9.15 . 5,5,7-trimethyl-4-(pyridin-4-yl)-3,4,5,6-tetrahydroquinaz oline-2-thiol (S V): Yield: 77\%; Colour: Yellow; m.p.: $278^{\circ} \mathrm{C}$; IR (KBr, cm $\left.{ }^{-1}\right): 1667$ ( $\left.\mathrm{Ar} \mathrm{C}=\mathrm{C}\right), 1467$ ( $\left.\mathrm{Ar} \mathrm{C}-\mathrm{C}\right), 3406$ $(\mathrm{N}-\mathrm{H}), 2853(\mathrm{Ar} \mathrm{C}-\mathrm{H}), 1367\left(\mathrm{CH}_{3}\right), 1599(\mathrm{C}=\mathrm{N}), 1254(\mathrm{C}-\mathrm{S})$; ${ }^{1} \mathrm{H}$ NMR (DMSO): $1.26 \delta\left(6 \mathrm{H}, \mathrm{s}, \mathrm{CH}_{3}\right), 1.82 \delta\left(3 \mathrm{H}, \mathrm{s}, \mathrm{CH}_{3}\right)$, $2.22 \delta\left(2 \mathrm{H}, \mathrm{s}, \mathrm{CH}_{2}\right), 4.19 \delta(1 \mathrm{H}, \mathrm{d}, \mathrm{N}-\mathrm{H}), 4.61 \delta(1 \mathrm{H}, \mathrm{d}, \mathrm{CH})$, $5.72 \delta(1 \mathrm{H}, \mathrm{s}, \mathrm{CH}), 6.05 \delta(1 \mathrm{H}, \mathrm{s}, \mathrm{S}-\mathrm{H}), 7.35 \delta(2 \mathrm{H}, \mathrm{d}, \mathrm{CH})$, $8.55 \delta(2 \mathrm{H}, \mathrm{d}, \mathrm{CH})$, DMSO $2.51 \delta \& 3.53 \delta ; \mathrm{MS}, \mathrm{m} / \mathrm{z}$ : 285.15[M+1]. Anal.: Calc. for C, 67.33; H, 6.71; N, 14.72; S, 11.23. Found: C, 67.28; H, 6.56; N, 14.51; S, 11.02.

\subsection{General Procedure for Synthesis of 4-Aryl-5, 5-Dimethyl-7-(2'Piperidin-1'-Yl-Ethyl)-2-Thiol-3,4, 5,6-Tetrahydroquinazolines (MB I-V)}

A mixture of 0.01 moles appropriate intermediate (S I-V), Piperidine \& formaldehyde in DMF were stirred for 2 hours in cooling conditions. Then the resulting reaction mixture was poured in ice bath. The obtain product (Scheme-IIa \& IIb) was filtered, dried \& recrystallized by $95 \%$ Ethanol.

5-(2-mercapto-5,5,7-trimethyl-3-(piperidin-1-ylmethyl)-3, 4, 5, 6 - tetrahydroquinazolin- 4- yl)-2-methoxyphenol (MB I): Yield: $82 \%$; Colour: Brown; m.p.: $292^{\circ} \mathrm{C}$; IR $\left(\mathrm{KBr}, \mathrm{cm}^{-1}\right)$ : $1652(\mathrm{Ar} \mathrm{C}=\mathrm{C}), 1417$ (Ar C-C), $2894($ Ar C-H), $1061(\mathrm{C}-\mathrm{N})$, $3426(\mathrm{~N}-\mathrm{H}), 3035(\mathrm{O}-\mathrm{H}), 1219$ (Ester C-O), $1314\left(\mathrm{CH}_{3}\right)$, $1265(\mathrm{C}-\mathrm{S}), 1600(\mathrm{C}=\mathrm{N}) ;{ }^{1} \mathrm{H}$ NMR (DMSO): $1.25 \delta(6 \mathrm{H}, \mathrm{s}$, $\left.\mathrm{CH}_{3}\right), 1.28 \delta\left(2 \mathrm{H}, \mathrm{m}, \mathrm{CH}_{2}\right), 1.53 \delta\left(4 \mathrm{H}, \mathrm{m}, \mathrm{CH}_{2}\right), 1.83 \delta(3 \mathrm{H}$, $\left.\mathrm{s}, \mathrm{CH}_{3}\right), 2.2 \delta\left(2 \mathrm{H}, \mathrm{s}, \mathrm{CH}_{2}\right), 2.45 \delta\left(4 \mathrm{H}, \mathrm{m}, \mathrm{CH}_{2}\right), 3.65 \delta(2 \mathrm{H}$, $\left.\mathrm{s}, \mathrm{CH}_{2}\right), 3.84 \delta\left(3 \mathrm{H}, \mathrm{s}, \mathrm{OCH}_{3}\right), 4.59 \delta(1 \mathrm{H}, \mathrm{d}, \mathrm{CH}), 5.33 \delta(1 \mathrm{H}$. $\mathrm{s}, \mathrm{OH}), 5.70 \delta(1 \mathrm{H}, \mathrm{s}, \mathrm{CH}), 6.32 \delta(1 \mathrm{H}, \mathrm{s}, \mathrm{S}-\mathrm{H}), 6.66 \delta(1 \mathrm{H}, \mathrm{d}$, $\mathrm{CH}), 6.69 \delta(1 \mathrm{H}, \mathrm{d}, \mathrm{CH}), 6.87 \delta(1 \mathrm{H}, \mathrm{s}, \mathrm{CH}), \mathrm{DMSO} 2.52 \delta \&$ $3.50 \delta ; \mathrm{MS}, \mathrm{m} / \mathrm{z}: 427.24[\mathrm{M}+1]$. Anal.: Calc. for $\mathrm{C}, 67.41 ; \mathrm{H}$, 7.78; N, 9.83; S, 7.50. Found: C, 67.22; H, 7.52; N, 9.60; S, 7.25 .

5,5,7-trimethyl-4-(3-nitrophenyl)-3-(piperidin-1-ylmethyl )-3,4,5,6-tetrahydroquinazoline-2-thiol (MB II): Yield: 87\%; Colour: Brown; m.p.: $>300^{\circ} \mathrm{C}$; IR $\left(\mathrm{KBr}, \mathrm{cm}^{-1}\right): 1664$ (Ar $\mathrm{C}=\mathrm{C}), 1464$ (Ar C-C), 2866 (Ar C-H), 1096 (C-N), 3404 $(\mathrm{N}-\mathrm{H}), 1528(\mathrm{~N}=\mathrm{O}), 1382\left(\mathrm{CH}_{3}\right), 1256(\mathrm{C}-\mathrm{S}), 1620(\mathrm{C}=\mathrm{N})$; ${ }^{1} \mathrm{H}$ NMR (DMSO): $1.25 \delta\left(6 \mathrm{H}, \mathrm{s}, \mathrm{CH}_{3}\right), 1.29 \delta\left(2 \mathrm{H}, \mathrm{m}, \mathrm{CH}_{2}\right)$, $1.52 \delta\left(4 \mathrm{H}, \mathrm{m}, \mathrm{CH}_{2}\right), 1.82 \delta\left(3 \mathrm{H}, \mathrm{s}, \mathrm{CH}_{3}\right), 2.0 \delta\left(2 \mathrm{H}, \mathrm{s}, \mathrm{CH}_{2}\right)$, $2.45 \delta\left(4 \mathrm{H}, \mathrm{m}, \mathrm{CH}_{2}\right), 3.60 \delta\left(2 \mathrm{H}, \mathrm{s}, \mathrm{CH}_{2}\right), 4.62 \delta(1 \mathrm{H}, \mathrm{d}, \mathrm{CH})$, $5.64 \delta(1 \mathrm{H}, \mathrm{s}, \mathrm{CH}), 5.84 \delta(1 \mathrm{H}, \mathrm{s}, \mathrm{S}-\mathrm{H}), 7.58 \delta(1 \mathrm{H}, \mathrm{d}, \mathrm{CH})$, $7.63 \delta(1 \mathrm{H}, \mathrm{d}, \mathrm{CH}), 8.09 \delta(1 \mathrm{H}, \mathrm{d}, \mathrm{CH}), 8.13 \delta(1 \mathrm{H}, \mathrm{s}, \mathrm{CH})$, DMSO $2.51 \delta \& 3.5 \delta ;$ MS, m/z: 426.21[M+1]. Anal.: Calc. for $\mathrm{C}, 64.76 ; \mathrm{H}, 7.09 ; \mathrm{N}, 13.13 ; \mathrm{S}, 7.52$. Found: C, 64.52; H, $6.89 ; \mathrm{N}, 12.96 ; \mathrm{S}, 7.36$.

5,5,7-trimethyl-4-phenyl-3-(piperidin-1-ylmethyl)-3,4,5,6 -tetrahydroquinazoline-2-thiol (MB III): Yield: 79\%; Colour: Brown; m.p.: $277^{\circ} \mathrm{C}$; IR $\left(\mathrm{KBr}, \mathrm{cm}^{-1}\right): 1660(\mathrm{Ar} \mathrm{C}=\mathrm{C}), 1423$ (Ar C-C), 2885 (Ar C-H), 1077 (C-N), $3420(\mathrm{~N}-\mathrm{H}), 1370$ $\left(\mathrm{CH}_{3}\right), 1248(\mathrm{C}-\mathrm{S}), 1596(\mathrm{C}=\mathrm{N}) ;{ }^{1} \mathrm{H}$ NMR (DMSO): $1.25 \delta$ $\left(6 \mathrm{H}, \mathrm{s}, \mathrm{CH}_{3}\right), 1.27 \delta\left(2 \mathrm{H}, \mathrm{m}, \mathrm{CH}_{2}\right), 1.53 \delta\left(4 \mathrm{H}, \mathrm{m}, \mathrm{CH}_{2}\right), 1.82$ $\delta\left(3 \mathrm{H}, \mathrm{s}, \mathrm{CH}_{3}\right), 2.19 \delta\left(2 \mathrm{H}, \mathrm{s}, \mathrm{CH}_{2}\right), 2.44 \delta\left(4 \mathrm{H}, \mathrm{m}, \mathrm{CH}_{2}\right), 3.63$ $\delta\left(2 \mathrm{H}, \mathrm{s}, \mathrm{CH}_{2}\right), 4.61 \delta(1 \mathrm{H}, \mathrm{d}, \mathrm{CH}), 5.7 \delta(1 \mathrm{H}, \mathrm{s}, \mathrm{CH}), 5.97 \delta$ $(1 \mathrm{H}, \mathrm{s}, \mathrm{S}-\mathrm{H}), 7.25 \delta(1 \mathrm{H}, \mathrm{t}, \mathrm{CH}), 7.27 \delta(2 \mathrm{H}, \mathrm{t}, \mathrm{CH}), 7.35 \delta$ $(2 \mathrm{H}, \mathrm{t}, \mathrm{CH}), \mathrm{DMSO} 2.5 \delta \& 3.51 \delta ; \mathrm{MS}, \mathrm{m} / \mathrm{z}: 381.22[\mathrm{M}+1]$. 
Anal.: Calc. for C, 72.40; H, 8.19; N, 11.01; S, 8.40. Found: C, 72.15; H, 8.09; N, 10.78; S, 8.19.

4-(3,4-dimethoxyphenyl)-5,5,7-trimethyl-3-(piperidin-1ylmethyl)-3,4,5,6-tetrahydroquinazoline-2-thiol (MB IV): Yield: $65 \%$; Colour: Orange; m.p.: $>300^{\circ} \mathrm{C}$; IR $\left(\mathrm{KBr}, \mathrm{cm}^{-1}\right)$ : $1646(\mathrm{Ar} \mathrm{C}=\mathrm{C}), 1425$ (Ar C-C), 2858 (Ar C-H), $1068(\mathrm{C}-\mathrm{N})$, $3443(\mathrm{~N}-\mathrm{H}), 1227$ (Ester C-O), 1360 (CH3), 1258 (C-S), $1629(\mathrm{C}=\mathrm{N}) ;{ }^{1} \mathrm{H}$ NMR (DMSO): $1.28 \delta\left(6 \mathrm{H}, \mathrm{s}, \mathrm{CH}_{3}\right), 1.53 \delta$ $\left(2 \mathrm{H}, \mathrm{m}, \mathrm{CH}_{2}\right), 1.59 \delta\left(4 \mathrm{H}, \mathrm{m}, \mathrm{CH}_{2}\right), 1.83 \delta(3 \mathrm{H}, \mathrm{s}, \mathrm{CH} 3), 2.21$ $\delta\left(2 \mathrm{H}, \mathrm{s}, \mathrm{CH}_{2}\right), 2.23 \delta\left(4 \mathrm{H}, \mathrm{m}, \mathrm{CH}_{2}\right), 3.61 \delta\left(2 \mathrm{H}, \mathrm{s}, \mathrm{CH}_{2}\right), 3.85$ $\delta\left(6 \mathrm{H}, \mathrm{s}, \mathrm{OCH}_{3}\right), 4.60 \delta(1 \mathrm{H}, \mathrm{d}, \mathrm{CH}), 5.22 \delta(1 \mathrm{H}, \mathrm{s}, \mathrm{CH}), 6.06$ $\delta(1 \mathrm{H}, \mathrm{s}, \mathrm{S}-\mathrm{H}), 6.67 \delta(1 \mathrm{H}, \mathrm{d}, \mathrm{CH}), 7.73 \delta(1 \mathrm{H}, \mathrm{d}, \mathrm{CH}), 7.76 \delta$ $(1 \mathrm{H}, \mathrm{s}, \mathrm{CH}), \mathrm{DMSO} 2.50 \delta \& 3.52 \delta ; \mathrm{MS}, \mathrm{m} / \mathrm{z}: 428.81[\mathrm{M}+1]$. Anal.: Calc. for C, 67.99; H, 7.99; N, 9.51; S, 7.26. Found: C, $67.65 ; \mathrm{H}, 7.72 ; \mathrm{N}, 9.26 ; \mathrm{S}, 7.01$.

5,5,7-trimethyl-3-(piperidin-1-ylmethyl)-4-(pyridin-4-yl)3,4,5,6-tetrahydroquinazoline-2-thiol (MB V): Yield: 78\%; Colour: Yellow; m.p.: $268^{\circ} \mathrm{C}$; IR $\left(\mathrm{KBr}, \mathrm{cm}^{-1}\right): 1653(\mathrm{Ar}$ $\mathrm{C}=\mathrm{C}), 1418(\mathrm{Ar} \mathrm{C}-\mathrm{C}), 2894(\mathrm{Ar} \mathrm{C}-\mathrm{H}), 1094(\mathrm{C}-\mathrm{N}), 3441$ $(\mathrm{N}-\mathrm{H}), 1368(\mathrm{CH} 3), 1265(\mathrm{C}-\mathrm{S}), 1600(\mathrm{C}=\mathrm{N}) ;{ }^{1} \mathrm{H}$ NMR (DMSO): $1.25 \delta\left(6 \mathrm{H}, \mathrm{s}, \mathrm{CH}_{3}\right), 1.28 \delta\left(2 \mathrm{H}, \mathrm{m}, \mathrm{CH}_{2}\right), 1.53 \delta$ $\left(4 \mathrm{H}, \mathrm{m}, \mathrm{CH}_{2}\right), 1.8 \delta\left(3 \mathrm{H}, \mathrm{s}, \mathrm{CH}_{3}\right), 2.21 \delta\left(2 \mathrm{H}, \mathrm{s}, \mathrm{CH}_{2}\right), 2.44 \delta$ $\left(4 \mathrm{H}, \mathrm{m}, \mathrm{CH}_{2}\right), 3.62 \delta\left(2 \mathrm{H}, \mathrm{s}, \mathrm{CH}_{2}\right), 4.59 \delta(1 \mathrm{H}, \mathrm{d}, \mathrm{CH}), 5.71 \delta$ $(1 \mathrm{H}, \mathrm{s}, \mathrm{CH}), 6.08 \delta(1 \mathrm{H}, \mathrm{s}, \mathrm{S}-\mathrm{H}), 7.36 \delta(2 \mathrm{H}, \mathrm{d}, \mathrm{CH}), 8.54 \delta$ (2H, d, CH), DMSO $2.52 \delta \& 3.51 \delta ;$ MS, m/z: 382.22[M+1]. Anal.: Calc. for C, 69.07; H, 7.90; N, 14.65; S, 8.38. Found: C, 68.92; H, 7.76; N, 14.49; S, 8.13.

\subsection{Antibacterial Study}

Culture of microorganisms: Bacterial samples used for primary screening of antibacterial activity for the compounds. Bacteria strains were supplied from ARIBAS, namely Bacillus subtilis, Pseudomonas aurigenosa, Escherichia coli. They were maintained by periodical transfer on fresh nutrie nt agar slant.

Preparation of media: The antibacterial activities were carried out by using Mueller-Hinton agar plate. The composition of the medium was Nutrient broth $13 \mathrm{~g} \&$ Agar-Agar powder $30 \mathrm{~g}$. Both ingredients were completely dissolved in $1 \mathrm{~L}$ of distilled water \& the $\mathrm{pH}$ of the medium was adjusted to $7.4 \mathrm{pH}$. The medium was sterilized in an autoclave at $121^{\circ} \mathrm{C}$ for $15 \mathrm{~min}$. It was then cooled down to $45^{\circ} \mathrm{C} \& 20 \mathrm{ml}$ was poured in each sterilized Petri dish.

Preparation of inoculums: A fresh microbial seed was prepared separately by sub culturing in to nutrient broth medium and incubated at $37^{\circ} \mathrm{C}$.

Antibacterial screening test: The antibacterial activity was performed after the checking of minimum inhibitory concentration (MIC) by taking $(0.04 \mathrm{~g} / \mathrm{mL})$ of sample using the cup method. Minimum inhibitory concentration is the lowest substance concentration at which no sign of bacterial growth was detectable microscopically. The test compounds were dissolved in DMSO to produce $(0.04 \mathrm{~g} / \mathrm{mL})$ for minimum inhibitory concentration (MIC). Different compounds on different microorganisms were placed in each cup. The plates were first placed in a refrigerator for 30 minutes and incubated at $37^{\circ} \mathrm{C}$ for 24 hours. The results were recorded by measuring the zone of inhibition in the plate and recorded for further calculation.

\section{Conclusions}

The antibacterial; activity of the compound MB I to MB V was screened against the three different types of bacterial strains (P. aurigenosa, E. coli, B. subtilis). None of these compound was found to be effective against $P$. aurigenosa However all these compounds are active against $B$. subtilis and $E$. coli compare to standard i.e. ciprofloxacin, the compound reported here are comparable against E. coli. However, all the compounds have very good activity against the bacterial strain, it is interesting to note that MB I and MB II are the most potent agents, have $\mathrm{OH}$ and $\mathrm{NO}_{2}$ group attached to aromatic ring respectively.

\section{ACKNOWLEDGEMENTS}

We thank Charutar Vidya Mandal, New Vallabh Vidyana gar for providing facility and financial support to undertake the research work. The authors are also gratefully acknowle dged the support of Torrent pharmaceuticals Ltd Research centre, Ahmedabad, Gujarat, India.

\section{REFERENCES}

[1] Amin K M, Kamel M M, and Anwar M M. European Journal of Medicinal Chemistry. 45, 2117-2131, 2010.

[2] Alagarsamy V, Raja Solomon V, Dhanabal K. Bioorganic \& Medicinal Chemistry. 15, 235-241, 2007.

[3] Vijaynathappa J, Bhojraj S. Journal of health sciences. 54, 524-528, 2008.

[4] Gaur K, Kori M.L. Academic J. of Plant Sci. 2, 60-64, 2009.

[5] Kerri, Rao M N A. Oxygen radical scavenging activity of curcumin, Int.J.pharma. 58, 237-240, 1990.

[6] Manihandrika P, Sridhar V. Indian journal of chemistry. 48B, 840-847, 2009.

[7] Al-Obaid A M, Abdel-Hamide S G, El-Kashef H A. European Journal of Medicinal Chemistry. 44, 2379-2391, 2009.

[8] Chandregowda V, Kush A.K, Chandrasekara Reddy G. European Journal of Medicinal Chemistry. 44, 3046-55, 2009.

[9] Giri R S, Thaker H M, Giordano T. Bioorganic \& Medicinal Chemistry. 18, 2796-2808, 2010.

[10] Qian L, Shen Y, Chen J. Acta Phys. -Chim. Sin. 22, 1372-1376, 2006.

[11] Giri R S, Thaker H M, Giordano T. European Journal of Medicinal Chemistry. 44, 2184-2189, 2009. 
[12] Laddha S S, Bhatnagar S P. Bioorg. \& Med. Chem.. 17, 6796-02, 2009.

[13] Jatav V, Mishra P, Kashaw S, Stables J P. European Journal of Medicinal Chemistry. 43, 1945-1954, 2008.

[14] Vachala D, Unnissa H. Indian journal of heterocyclic chemistry. 17, 347-350, 2008.

[15] Guang-Fang Xu, Bao-An Song, Bhadury P S. Bioo ganic \& Medicinal Chemistry. 15, 3768-3774, 2007.

[16] Kabri Y, Nadine A, Dume tre A L. European Journa of Medicinal Chemistry. 45, 616-622, 2010.

[17] Subramaniam A, Faaleolea E R, Goldman R C, Tuberculosis. 89, 334-353, 2009.

[18] Ashraf A. Khalil, Sami G. Abdel Hamide, Abdulrahman M. Al-Obaid, Hussein I. El-Subbagh; Substituted Quinazolines,
Part 2. Synthesis and In-Vitro Anticancer Evaluation of New 2-Substituted Mercapto-3H-quinazoline Analogs; Arch. Pharm. Pharm. Med. Chem., 2, 95-103, 2003.

[19] Guan J, Zhang Q, O'Neil M, Obaldia N 3rd, Ager A, Gerena L, Lin AJ. Antimalarial activities of new pyrrolo[3, 2-f] quinazoline-1, 3-diamine derivatives. Antimicrob Agents Chemother. Dec; 49(12):4928-33, 2005.

[20] Nakamoto K. IR of inorganic and coordination compound, John Willey \& Sons, pp 54, 1963.

[21] Honkanen E, Pipuri A, Kairisalo P, Nore P, Karppaness H and Paakari I, J. Med. Chem.; 26; 143, 1983.

[22] Saundane A. Rudresh K. Satyanarayan N. Hiremath S. Pharmacological screening of $6 \mathrm{H}, 11 \mathrm{H}$-Indolo $\{3,2-\mathrm{C}\}$ isoquinolin-5-ones \& their derivatives. J Ind pharm sci., 60, 379-383, 1998. 